\title{
Management of coeliac disease: a changing diagnostic approach but what value in follow up?
}

\author{
Monica Acalovschi, V Jayanthi, C S J Probert, J F Mayberry
}

\begin{abstract}
Objective - To assess the management of patients with coeliac disease in relation to a change in diagnostic method from jejunal suction biopsy to endoscopic biopsy.
\end{abstract}

Design - 16 item questionnaire survey of consultant members of the British Society of Gastroenterology.

Subjects - 359 consultant physician and gastroenterologist members of the society.

Main measures - Type of routine biopsy; repeat biopsy after gluten withdrawal; gluten rechallenge; follow up measurements; screening for malignancy; and methods of follow up, including special clinics.

Results - 270(70\%) members replied; $216(80 \%)$ diagnosed coeliac disease routinely by endoscopic duodenal biopsy, $30(11 \%)$ by jejunal capsule biopsy, and the remainder by either method. Only $156(58 \%)$ repeated the biopsy after gluten withdrawal, though more did so for duodenal than jejunal biopsies (134/216, $62 \% v 13 / 30,43 \% ; p<0.02)$. Follow up biopsies featured more duodenal than jejunal biopsies $(133 / 156,82 \%$ v 23/156, $15 \%$; $<0.02$ ). Regular follow up included assessments of weight $(259,96 \%)$ and full blood count $(238,88 \%)$ but limited assessment of serum B-12 and folate $(120,44 \%)$ and calcium $(105,39 \%)$ concentrations. Routine screening for malignancy is not performed, and there are few specialist clinics. $171(63 \%)$ respondents thought that patients should be followed up by a hospital specialist and $58(21 \%)$ by family doctors.

Conclusions - The practice of diagnosing coeliac disease varies appreciably from that in many standard texts. Many patients could be effectively cared for by their family doctor.

Implications - The British Society of Gastroenterology should support such management by family doctors by providing clear guidelines for them.

\section{Introduction}

Coeliac disease is a relatively common condition in western Europe, its prevalence being 50-74/100 000 in the United Kingdom and between 50 and $250 / 100000$ in other Western countries. ${ }^{2}$ A changing pattern of coeliac disease has been noticed both in adults and in children. Many patients have only mild symptoms and no signs of malabsorption. Presently, only $12 \%$ of adults with coeliac disease have overt malabsorption, compared with $63 \%$ when the technique of jejunal biopsy was first introduced 30 years ago. This changing pattern is obviously due to an improved diagnostic approach. As a consequence of the increased use and easy performance of upper gastrointestinal endoscopy with duodenal biopsy coeliac disease is now diagnosed in asymptomatic people or those with only minor symptoms. Increased awareness of the disease might be responsible for an apparent increase in its prevalence but also leads to earlier diagnosis and hence a decline in severity at diagnosis. Ten years ago the prevalence of members of the Coeliac Society in England and Wales correlated well with regional sales of jejunal biopsy capsules. ${ }^{4}$ Jejunal suction biopsies have been progressively replaced by duodenal endoscopic biopsies. It is now accepted that the diagnostic adequacy of multiple specimens taken through an endoscope is comparable to or even better than that of specimens taken with suction capsules. ${ }^{5-7}$ The advantages of endoscopic biopsies are their easy and rapid performance as well as visualisation of the loss of duodenal folds, a finding suggestive of coeliac disease which has $88 \%$ sensitivity and $83 \%$ specificity.

As the clinical picture is only rarely classic and histological abnormalities are characteristic but not always pathognomonic a definite diagnosis should be based on histological examination before and after a gluten free diet. ${ }^{2,89}$ A gluten free diet can improve the histological appearance within a few weeks, but normalisation of the mucosa may take from a few months to up to one to two years ${ }^{2}$; it is now accepted that gluten rechallenge to confirm coeliac disease is not mandatory in adults ${ }^{\circ}$ whereas it is regarded as standard practice in children. ${ }^{10}$ The recently introduced rectal gluten challenge is a single, safe, and reliable test which could become a welcome alternative to oral gluten challenge. ${ }^{11}$

Up to $14 \%$ of patients with coeliac disease may develop malignancy, ${ }^{8} 1213$ but there is no pattern of haematological or biochemical abnormality which enables lymphoma or other associated tumours to be diagnosed easily and clearly. The role of routine annual faecal 
occult blood testing which has been used to screen healthy populations for early tumours has yet to be evaluated in coeliac disease.

\section{Methods}

To assess the current management in Britain of adults with coeliac disease a 16 item questionnaire (box) was sent to the 359 members of the British Society of Gastroenterology, who were either consultant physicians or gastroenterologists. More junior doctors, of senior registrar grade or lower, were excluded. Subjects were asked to indicate which alternative corresponded most closely to their clinical practice; no response was a third option.

\begin{abstract}
Results
Two hundred and seventy completed questionnaires were received (response rate $75 \%$ ). The specialty of the respondents was medicine $103(38 \%)$ and gastroenterology $46(17 \%)$; no comment was given in 121 cases (45\%). Most doctors (151, 56\%) cared for between 10 and 49 patients with coeliac disease, although $76(28 \%)$ managed more than 50 .

Two hundred and sixteen respondents $(80 \%)$ diagnosed coeliac disease by endoscopic duodenal biopsy; only $30(11 \%)$
\end{abstract}

\section{Coeliac disease (CD): its diagnosis and management}

Specialty: medicine/gastroenterology (please specify)

Approximate number of patients with coeliac disease (CD) under your care?
0-9
$10-49$
50-99
$\geqslant 100$

\section{Diagnosis and management:}

1 What type of biopsies do you routinely take? Endoscopic duodenal/jejunal capsule biopsy

2 Do you routinely perform a small bowel radiological examination?

3 Do you routinely repeat small bowel/duodenal biopsy after gluten withdrawal? If yes when?

4 Do you routinely perform a gluten rechallenge test?

5 Do you follow up patients with $\mathrm{CD}$ by tests?

6 Do you have computerised follow up for checking:
(1) calcium
(2) folic acid
(3) anaemia?

7 Which of the following do you perform each time you review a patient (please circle)?

Weight/full blood count/B-12 and folic acid concentrations/ liver function tests including calcium/faecal occult blood test

8 Do you have a screening system to detect malignant change?

9 Do you have a separate clinic for patients with CD?

10 Do you have a specialist nurse for patients with $C D$ ?

11 Do you have an open access clinic for CD?

12 Do you encourage membership of self help groups?

13 Do you make use of information booklets for your patients with $\mathrm{CD}$ ?

14 Do you make use of information videos for your patients with $\mathrm{CD}$ ?

15 Do you refer patients with $\mathrm{CD}$ to fellow (consenting) patients to discuss problems?

16 Do you think, under the new contract system, patients should be followed up by their general practitioners rather than by a hospital specialist? continued to use jejunal capsule biopsy as their technique of choice. The remaining $24(9 \%)$ used either method depending on the patient and the facilities available. One hundred and fifty six respondents (58\%) repeated the biopsy after gluten withdrawal: 98 doctors did so at six months and 33 after a year; the other 25 doctors had no fixed time for repeating the biopsy. Doctors who initially chose endoscopic biopsy to reach the diagnosis repeated the biopsy after gluten withdrawal significantly more often than those performing jejunal suction biopsy $(134 / 216,62 \% v 13 / 30$, $\left.43 \% ; \chi^{2}=8.6 ; \mathrm{p}<0.02\right)$. For follow up endoscopic biopsy was more commonly used than jejunal capsule biopsy $(133 / 156,82 \% v$ $\left.23 / 156,15 \% ; \chi^{2}=7.3 ; \mathrm{p}<0.02\right)$. Only seven doctors would consider rechallenging their patients with gluten once they were in remission, mainly in the case of patients who were children or for research. Assessment of the small bowel by radiological examination was performed by $67(25 \%)$ consultant members of the society.

Patients with coeliac disease are regularly followed up with body weight assessment (259, 96\%) and haematological and biochemical reviews. Two hundred and thirty eight doctors (88\%) asked for routine full blood counts and $120(44 \%)$ for measurement of serum B-12 and folate concentrations. Liver function tests and calcium estimations were regularly performed by $105(39 \%)$ of respondents. There was no routine screening for malignancy, except by annual clinical review in 13 clinics $(5 \%)$ or by carcinoembryonic antigen in three centres $(1 \%)$.

The last eight items of the questionnaire referred to methods of patient follow up. As a rule there was no separate clinic for patients with coeliac disease (13(5\%) exceptions) and only $75(28 \%)$ of doctors offered an open access service. Only one respondent had a specialist nurse for patients with coeliac disease but almost all (96\%) encouraged membership of self help groups and used information booklets for these patients (248, $92 \%)$. Only a few doctors $(18,7 \%)$ made use of information videos for their patients, but almost a third (79) referred patients to fellow sufferers to discuss mutual problems.

The last question dealt with the future role of general practitioners in long term care. One hundred and seventy one consultants (63\%) considered that patients with coeliac disease should be followed up by a hospital specialist and $58(21 \%)$ by general practitioners; others $(13,5 \%)$ thought that this decision depended on the general practitioner and that it should be possible to develop guidelines for referral to hospital in that situation.

\section{Discussion}

During the past five years there has been a dramatic change in the standard practice for diagnosing coeliac disease. Whereas the traditional approach had been to use jejunal biopsies, which were often unpleasant for the patient, time consuming, and required radiological screening, widespread recognition 
of the value of endoscopic duodenal biopsies has led to a much more rapid procedure which has greater patient acceptability. In experienced hands endoscopic biopsy can take less than 10 minutes compared with 30 minutes or more commonly required for jejunal suction biopsies. Such a significant diagnostic change will probably influence the incidence of diagnosis in adults; endoscopy in childen requires a general anaesthetic and carries less advantage. Despite these comments the incidence of coeliac disease in adults has remained fairly constant during the past decade. With the introduction of open access endoscopy to which family doctors can directly refer patients, the diagnosis of coeliac disease has been opened up to those other than specialist gastroenterologists. With this in mind, how important is the long term care of patients with this condition by such specialists? One aspect of this survey was an attempt to define the role of gastroenterologists in long term care. If they have a role it must be in the early detection of biochemical and haemotological abnormalities or of malignant change. These approaches include measurement of serum folate and calcium concentrations and are well established. Coeliac disease predisposes the sufferer to both gastrointestinal lymphomas and oesophageal cancer. ${ }^{14}$ All intestinal neoplasms tend to bleed microscopically, and faecal occult blood testing can detect abnormalities of this variety, although not specifying their nature. The role of faecal occult blood testing in detecting neoplasia in coeliac disease has not been investigated. Its role in the early detection of colorectal and other gastrointestinal cancers in healthy populations, however, is clear and well established. Compliance is often a problem in these groups, although less so in groups at increased risk of cancer, such as siblings or patients with a history of cancer. We wished to see if such an approach had been adopted by any gastroenterologists working with patients with coeliac disease. In practice, less than half the consultants in the United Kingdom routinely monitored these blood tests and less than $10 \%$ had any cancer or lymphoma detection programme.

Although diagnostic practice in coeliac disease has changed significantly, there has not been a comparable change in management. The two main aspects of this study were who should provide continuing care and the possible role of early detection of neoplastic change. Body weight assessment seemed to be the main objective assessment by consultants during routine follow up. Family doctors could probably provide as effective a service at less cost. Indeed, with their experience of immunisation programmes and health screening they might achieve more complete follow up and patient compliance. In the present climate of care profiles and resource management the justification for such a hospital based approach needs to be continually re-evaluated. Family doctors could perform these tests and be given guidelines to suitable indicators of a need for referral. Before such an approach is adopted, however, patients themselves should be approached to ascertain their wishes. Until now there has been only limited acknowledgement by hospital practitioners of the social needs of such patients. ${ }^{15}$ This is well exemplified by the fact that only one clinic provided a nurse based counselling service.

The time seems to have come for the British Society of Gastroenterology to produce guidelines for the management of coeliac disease, which could be made available to non-specialists for use in general practice. These guidelines should identify which variables should be regularly reviewed and indicate when patients should again be referred to hospital.

A responsible family doctor could probably manage a patient with coeliac disease, with a significant cost saving without any deterioration in care. This, of course, contrasts with the current practice of most gastroenterologists in Britain.

Dr Monica Acalovschi was a visiting fellow of the Scotland Romania Medicaid at Leicester General Hospital.

1 Logan RFA, Rifkind EA, Busultil A, Gilmour HM. Prevalence and "incidence" of celiac disease in Edinburgh and the Lothian region of Scotland. Gastroenterology 1986;90:334-42.

2 Trier JS. Celiac sprue. In: eds. Sleisenger MH, Fordtran JS, Gastrointestinal disease, pathophysiology, diagnosis, management 4th ed, Vol 2. WB Saunders, 1989: 1134-52.

3 Logan RFA, Tucker G, Rifkind EA, Heading RC, Ferguson A. Changes in clinical features of coeliac disease in adults in Edinburgh and the Lothians 1960-79. BMF 1983;286:95-7.

4 Swinson CM, Levi AJ. Is coeliac disease underdiagnosed? BMF 1980;281:1252-60.

5 Brocchi E, Coazza GR, Caletti G, Treggiari AE, Barbara L, Gasbarini G. Endoscopic demonstration of loss of L, Gasbarini G. Endoscopic demonstration of loss of duodenal folds in the diagn
Engl $\mathcal{F}$ Med 1988;319:741-4.

6 Gillberg R, Ahren C. Coeliac disease diagnosed by means of duodenoscopy and endoscopic duodenal biopsy. Scand f Gastroenterol 1977;12:911-6.

7 Mee AS, Burke N, Vallon AG, Newman J, Cotton PB. Small bowel biopsy for malabsorption: comparison of the diagnostic accuracy of endoscopic forceps and capsule biopsy specimens. BMF 1985;295:769-72

8 Shearman DJ, Finlayson NDC, eds. Coeliac disease and other mucosal diseases. In: Diseases of the gastrointestinal tract and the liver. 11th ed.:Churchill Livingstone, $1989 ; 407-58$

9 Collin P, Hallstrom O, Naki N, Vlandere N, Kevvilainen O. Atypical coeliac disease found with serological O. Atypical coeliac disease found with sero

10 Kumar PJ, O'Donoghue DP, Stenson K, Dawson AN. Reintroduction of gluten in adults and children with Reintroduction of gluten in adults and child

11 Loft DE, Narsh NN, Crowe PT. Rectal gluten challenge and diagnosis of coeliac disease. Lancet 1990; 335: $1293-5$.

12 Swinson CN, Slavin G, Coles EC, Booth CC. Coeliac disease and malignancy. Lancet 1983;i:111-5.

13 Freeman HJ, Chui BK. Small bowel malignant lymphoma complicating celiac disease sprue and the mesenteric cavitation syndrome. Gastroenterology 1986;90:2008-12.

14 Holmes GKT, Prior P, Lane MR, Pope D, Allan RN. Malignancy in coeliac disease - effect of a gluten free diet. Gut 1989;30:333-8.

15 Growing up with coeliac disease [Editorial]. Lancet 1988;ii: $1230-5$ 